\title{
Gap junctions and connexin hemichannels in the regulation of haemostasis and thrombosis
}

Article

Accepted Version

Vaiyapuri, S., Flora, G. D. and Gibbins, J. M. (2015) Gap junctions and connexin hemichannels in the regulation of haemostasis and thrombosis. Biochemical Society Transactions, 43 (3). pp. 489-494. ISSN 1470-8752 doi: https://doi.org/10.1042/BST20150055 Available at https://centaur.reading.ac.uk/40338/

It is advisable to refer to the publisher's version if you intend to cite from the work. See Guidance on citing.

Published version at: http://www.biochemsoctrans.org/bst/043/bst0430489.htm

To link to this article DOI: http://dx.doi.org/10.1042/BST20150055

Publisher: Portland Press

All outputs in CentAUR are protected by Intellectual Property Rights law, including copyright law. Copyright and IPR is retained by the creators or other copyright holders. Terms and conditions for use of this material are defined in the End User Agreement.

www.reading.ac.uk/centaur 
Central Archive at the University of Reading

Reading's research outputs online 


\title{
Gap junctions and connexin hemichannels in the regulation of haemostasis and thrombosis
}

\author{
Sakthivel Vaiyapuri ${ }^{1,2}$, Gagan D. Flora ${ }^{1}$ and Jonathan M. Gibbins ${ }^{1 \#}$
}

${ }^{1}$ Institute for Cardiovascular and Metabolic Research, School of Biological Sciences and ${ }^{2}$ School of Pharmacy, University of Reading, Reading, UK.

\#Correspondence author: Professor Jonathan Gibbins, Institute for Cardiovascular and Metabolic Research, School of Biological Sciences, Harborne Building, University of Reading, Reading, UK. Tel: +44 118378 7082; Email: j.m.gibbins@,reading.ac.uk

\begin{abstract}
Platelets are involved in the maintenance of haemostasis but their inappropriate activation leads to thrombosis, a principal trigger for heart attack and ischemic stroke. Although platelets circulate in isolation, upon activation they accumulate or aggregate together to form a thrombus, where they function in a coordinated manner to prevent loss of blood and control wound repair. Recent reports indicate that the stability and functions of a thrombus are maintained through sustained, contact dependent signalling between platelets. Given the role of gap junctions in the coordination of tissue responses, it was hypothesized that gap junctions may be present within a thrombus and mediate intercellular communication between platelets. Therefore studies were performed to explore the presence and functions of connexins in platelets. In this brief review, the roles of hemichannels and gap junctions in the control of thrombosis and haemostasis and the future directions for this research will be discussed.
\end{abstract}

Key words: Platelets; intercellular communication; contact-dependent signalling; connexin; thrombosis; haemostasis

\section{Introduction}

Platelets are small circulating blood cells that play paramount roles in the formation of a blood clot upon injury in order to prevent bleeding [1]. However, the inappropriate activation of platelets in the arterial circulation under pathological conditions such as the rupture of atherosclerotic lesions leads to the formation of blood clots within blood vessels which restrict blood flow to downstream organs (such as heart and brain). The occlusion of coronary arteries leads to myocardial infarction and thrombosis in the cerebral vasculature, to ischemic stroke $[1,2]$. While targeting platelets as an antithrombotic strategy is proven to reduce the risk of thrombosis, currently available anti-platelet approaches are unrefined, ineffective in certain patients and cause serious side effects such as bleeding [3]. A key focus of research to understand the mechanisms of platelet function is therefore aimed at the development of more effective and safer anti-platelet approaches to prevent or treat thrombosis. A number of well-orchestrated signalling events control the activation of circulating platelets to form multicellular platelet aggregates (thrombus) [1]. Activation of platelets leads to fibrinogen binding to integrin $\alpha \mathrm{IIb} \beta 3$ on the surface of platelets, which bridges these cells together and maintains sustained contact-dependent signalling between them $[1,4,5]$. Other surface receptors such as Eph kinases and their ligands, ephrins have been reported to play major roles in the regulation of contact-dependent signalling between platelets within a thrombus, and thereby contributing to thrombus growth, stability and potentially resolution [6-10].

Connexins are a family of transmembrane proteins that are expressed widely with over 20 connexins identified in various mammalian cells $[11,12]$. They are usually named according to native molecular mass, for example connexin $37(\mathrm{Cx} 37)$ has a molecular mass of $37 \mathrm{kDa}$ [13]. Six connexin monomers oligomerise to form a cylinder-like structure called a connexon or hemichannel (Figure 1). Following the formation of hemichannels in the endoplasmic reticulum, they are transported to the plasma membrane through a vesicular transport system where they act as a conduit to transfer signalling molecules between the cytoplasm and extracellular regions [14]. The association of cells which contain connexins leads to docking of opposing hemichannels to form tightly controlled pore structures, namely gap junctions (Figure 1). Gap junctions have a pore size of 2-3nm and enable the direct exchange of signalling molecules of up to approximately $1 \mathrm{kDa}$ between the cytoplasm of adjacent cells [11]. The signalling molecules that are transported through these channels vary based 
on the properties of specific connexins and the external signalling events that control their function $[11,15]$. Several gap junctions assemble together to form gap junction plaques at the interface between two cells. As the gap junction plaques between cells are tightly regulated to avoid any leakage during molecular transport, they may also play roles in maintaining cellular adhesions [11].

A key role of gap junctions is to enable rapid and coordinated responses between specialised cells within a tissue. Although the functions of hemichannels and gap junctions are well studied in tissues where sustained cell-cell interactions occur, such as cardiac myocytes [16], arterial endothelial cells [17], oocytes [18], neuronal [19] and bone marrow stromal cells [20, 21], the concept of their roles in circulating cells is relatively recent. Studies in this area were initiated by ground breaking research that revealed the roles for hemichannels and gap junctions in circulating leukocytes such as monocytes and T-cells [22-25]. While platelets circulate in isolation under normal conditions, as described, upon activation they come into close proximity to form a thrombus within which prolonged platelet-platelet contact occurs [7]. Therefore the hypothesis that platelets may possess connexins and form gap junctions to maintain sustained interactions and signalling between platelets within thrombi was tested.

\section{Connexins in platelets}

Since platelets are derived from megakaryocytes in the bone marrow, we sought to analyse the transcript levels for various connexins in these cells. The analysis revealed the presence of multiple connexins in megakaryocytes with notable levels of Cx37, Cx40 and Cx62 [26]. Although Cx37 and Cx40 have been well characterised in other cell types, Cx62 is a relatively newly identified family member and much remains unknown about this protein. The expression (at the protein level) of multiple connexins (Cx37, $\mathrm{Cx} 40, \mathrm{Cx} 43$ and $\mathrm{Cx} 32)$ in platelets was subsequently confirmed using a number of methods [26, 27]. The presence of connexins in megakaryocytes and their roles in mediating the interactions with stromal cells and platelet production have also been previously reported [20, 28-30].

\section{Gap junctions between platelets}

To explore whether platelet connexins form gap junctions, the interactions of platelets within thrombi were analysed by transmission electron microscopy. Gap junction-like structures at the regions where cells interact were evident (Figure 2) [26]. To further confirm the presence of gap junctions by functional analysis, fluorescence recovery after photo bleaching analysis (FRAP) was performed in thrombi. FRAP is a microscopy-assisted technique which enables the tracking of fluorescent dyes that are permeable through gap junctions between cells. The recovery of fluorescence following bleaching within a cell or a region of tissue can be quantified by acquiring images at a high speed. The migration of a fluorescent dye, calcein-AM was studied after photo bleaching within thrombi that were formed under flow using human blood on a collagen-coated surface. Direct intercellular communication between platelets was detected by FRAP and this phenomenon was inhibited by broad spectrum gap junction blockers such as carbenoxolone and 18ß-glycyrrhetinic acid, and $\mathrm{Cx} 37$ and Cx43 selective mimetic peptide, ${ }^{37,43}$ Gap27 [26]. Angelillo-Scherrer et al. [27] used an alternative approach to demonstrate intercellular communication where neuro biotin was microinjected into platelets within aggregates and was shown to migrate into other cells. These experiments provided strong evidence to demonstrate the gap junction mediated intercellular communication (GJIC) within platelets.

\section{Role of hemichannels during the activation of platelets}

Upon injury, sub-endothelial collagen is exposed and platelets are recruited to the damaged site where they bind to collagen and form a monolayer of cells. Subsequently they become activated and release their granule contents such as ADP and simultaneously synthesise and release thromboxane $A_{2}$. These molecules together with thrombin, which is produced on the surface of platelets, activate more platelets and recruit them to form a growing thrombus [1]. Therefore initial experiments were performed to explore the roles for connexins in platelet activation.

Fibrinogen binding to integrin $\alpha \operatorname{IIb} \beta 3$ and P-selectin exposure (a marker for $\alpha$-granule secretion) were measured using flow cytometry by gating individual platelets. The addition of gap junction blockers 
such as carbenoxolone and $18 \beta$-glycyrrhetinic acid and connexin mimetic peptides such as ${ }^{37,43}$ Gap 27 and ${ }^{40} \mathrm{Gap} 27$ (which targets $\mathrm{Cx} 40$ ) reduced substantially the levels of fibrinogen binding and Pselectin exposure in human platelets upon activation with agonists and, similar results were obtained using platelets isolated from $\mathrm{Cx} 37$ and $\mathrm{Cx} 40$ deficient mice [26, 31]. Moreover, calcium mobilisation measured in platelets under the conditions which disfavour aggregation was also reduced by these inhibitors [26]. These results revealed the importance of connexin hemichannels on platelets during initial activation prior to establishing platelet-platelet contact.

\section{Role of gap junctions within thrombi}

To assess the potential role of gap junctions within thrombi, thrombus formation assays were performed in microfluidic flow cells under arterial flow conditions. Thrombi were allowed to form over a period of time in flow chambers using fluorescently labelled human blood and their growth, size and stability were monitored by microscopy. The addition of connexin inhibitors reduced substantially thrombus formation under flow conditions indicating that gap junctions are important for this process [26].

Following fibrin clot formation, a consequence of the activation of coagulation pathways, clot retraction occurs to facilitate wound repair. This event is controlled by platelet outside-in signalling transduced by integrin $\alpha \mathrm{IIb} \beta 3$ [5]. For this event to occur, the platelets must contract in a coordinated manner towards the centre of the clot. This phenomenon can be readily observed in vitro. To understand the importance of gap junctions during this process, clot retraction assays were performed using platelet-rich plasma from human (in the presence and absence of gap junction blockers and connexin mimetic peptides) and $\mathrm{Cx} 37$ or $\mathrm{Cx} 40$ deficient mice. In the absence of connexin function, the clot retraction was reduced substantially which strongly supports the notion that gap junctions are involved in the control of clot retraction process [26, 31].

\section{Connexins in the control of thrombosis and haemostasis}

Since the thrombus formation measured under in vitro conditions using human blood was inhibited substantially by gap junction blockers, the effect of connexin mimetic peptide $\left({ }^{37,43} \mathrm{Gap} 27\right)$ in thrombosis in vivo was explored. Following infusion of ${ }^{37,43} \mathrm{Gap} 27$ mimetic peptide along with a fluorescently conjugated platelet labelling antibody (anti-GPIb), thrombus formation was induced in cremaster arterioles of anaesthetised mice by laser injury and the thrombus growth, size and stability were monitored by an intravital microscopy. The resulting data demonstrated the ability of ${ }^{37,43} \mathrm{Gap} 27$ to reduce thrombosis suggesting that gap junctions perform important roles in the regulation of thrombus formation and stability (Figure 3) [26].

To further understand the role of connexins in the maintenance of haemostasis, tail bleeding was assessed in mice in the presence and absence of ${ }^{37,43} \mathrm{Gap} 27$ and ${ }^{40} \mathrm{Gap} 27$. Mice treated with these connexin mimetic peptides showed increased bleeding compared to controls indicating that connexin hemichannels and gap junctions are involved in the maintenance of haemostasis under physiological conditions [26, 31].

\section{Cx37 and Cx40 function independently from each other}

Connexins are able to form either homomeric or heteromeric hemichannels resulting in homotypic or heterotypic gap junctions. For example Cx26 and Cx32 together form heteromeric hemichannels and therefore, heterotypic gap junctions [32]. These different forms of hemichannels and gap junctions have differential conductance and regulation properties. Since the presence of multiple connexins was confirmed in platelets, the possibilities of them forming homomeric or heteromeric hemichannels were explored. Using the platelets from $\mathrm{Cx} 37$ or $\mathrm{Cx} 40$ deficient mice and selective mimetic peptide inhibitors for these connexins $\left({ }^{37,43} \mathrm{Gap} 27\right.$ and $\left.{ }^{40} \mathrm{Gap} 27\right), \mathrm{Cx} 37$ and $\mathrm{Cx} 40$ were found to function independently from each other [31]. There are, however, other connexin family members present in platelets. Therefore combinations within hemichannels and gap junctions cannot be excluded.

\section{Conclusions and future perspectives}

Initial research has provided compelling evidence for the roles of hemichannels and gap junctions in the regulation of thrombosis and haemostasis [26, 31]. Hemichannels are important during the initial 
activation of circulating platelets and later, gap junctions may modulate thrombus formation and stability. Hemichannels and gap junctions may allow the rapid transport of several signalling molecules such as calcium, inositol 1,4,5-trisphosphate (IP3) and cyclic nucleotides between platelets across a thrombus. While the hemichannels may facilitate the transport of molecules between platelets and the external environment, gap junctions may regulate the sustained signalling within the thrombus and control clot retraction.

As discussed, gap junctions are known to cluster as gap junction plaques on the surface of cells, maintaining cell-cell adhesion and synchronise the functions of adjacent cells [11]. Similarly, in platelets, gap junction plaques may be formed and contribute to cell-cell adhesion complementing the actions of additional adhesive receptors such as integrin $\alpha I I b \beta 3$, Eph kinases and ephrins. The role of gap junctions in the regulation of platelet adhesion has yet to be explored.

The regulation of connexin functions has been explored in several cell types such as heart [16] and brain [19]. The intracellular regions of connexins are known to interact with several cytoplasmic signalling proteins such as $\beta$ catenin, Nov, protein kinases, caveolin and Dbn1 and cytoskeletal proteins such as tubulin to regulate channel properties [33]. Further work is required to dissect the mechanisms that regulate the roles of hemichannels and gap junctions in platelets, and the nature of signalling molecules that are transported through these channels. Given the additional roles for platelets in inflammatory responses $[2,34,35]$, it is tempting to speculate that these structures in platelets may form a nexus of cellular interactions and signalling to control complex regulatory processes with a range of cell types.

\section{Funding}

The authors would like to thank the British Heart Foundation for funding this research.

\section{References}

1 Gibbins, J. M. (2004) Platelet adhesion signalling and the regulation of thrombus formation. Journal of cell science. 117, 3415-3425

2 Davi, G. and Patrono, C. (2007) Platelet activation and atherothrombosis. The New England journal of medicine. 357, 2482-2494

3 Barrett, N. E., Holbrook, L., Jones, S., Kaiser, W. J., Moraes, L. A., Rana, R., Sage, T., Stanley, R. G., Tucker, K. L., Wright, B. and Gibbins, J. M. (2008) Future innovations in anti-platelet therapies. British journal of pharmacology. 154, 918-939

4 Li, Z., Delaney, M. K., O'Brien, K. A. and Du, X. (2010) Signaling during platelet adhesion and activation. Arteriosclerosis, thrombosis, and vascular biology. 30, 2341-2349

5 Shattil, S. J. and Newman, P. J. (2004) Integrins: dynamic scaffolds for adhesion and signaling in platelets. Blood. 104, 1606-1615

6 Prevost, N., Woulfe, D., Tanaka, T. and Brass, L. F. (2002) Interactions between Eph kinases and ephrins provide a mechanism to support platelet aggregation once cell-to-cell contact has occurred. Proceedings of the National Academy of Sciences of the United States of America. 99, 9219-9224

7 Prevost, N., Woulfe, D., Tognolini, M. and Brass, L. F. (2003) Contact-dependent signaling during the late events of platelet activation. Journal of thrombosis and haemostasis : JTH. 1, 16131627

8 Prevost, N., Woulfe, D. S., Jiang, H., Stalker, T. J., Marchese, P., Ruggeri, Z. M. and Brass, L. F. (2005) Eph kinases and ephrins support thrombus growth and stability by regulating integrin outside-in signaling in platelets. Proceedings of the National Academy of Sciences of the United States of America. 102, 9820-9825

9 Prevost, N., Woulfe, D. S., Tognolini, M., Tanaka, T., Jian, W., Fortna, R. R., Jiang, H. and Brass, L. F. (2004) Signaling by ephrinB1 and Eph kinases in platelets promotes Rap1 activation, platelet adhesion, and aggregation via effector pathways that do not require phosphorylation of ephrinB1. Blood. 103, 1348-1355

10 Vaiyapuri, S., Sage, T., Rana, R. H., Schenk, M. P., Ali, M. S., Unsworth, A. J., Jones, C. I., Stainer, A. R., Kriek, N., Moraes, L. A. and Gibbins, J. M. (2015) EphB2 regulates contact-dependent and contact-independent signaling to control platelet function. Blood. 125, 720-730 
11 Goodenough, D. A. and Paul, D. L. (2009) Gap junctions. Cold Spring Harbor perspectives in biology. 1, a002576

12 Sohl, G. and Willecke, K. (2004) Gap junctions and the connexin protein family. Cardiovascular research. 62, 228-232

13 Sohl, G. and Willecke, K. (2003) An update on connexin genes and their nomenclature in mouse and man. Cell communication \& adhesion. 10, 173-180

14 Goodenough, D. A. and Paul, D. L. (2003) Beyond the gap: functions of unpaired connexon channels. Nature reviews. Molecular cell biology. 4, 285-294

15 Alves, L. A., Nihei, O. K., Fonseca, P. C., Carvalho, A. C. and Savino, W. (2000) Gap junction modulation by extracellular signaling molecules: the thymus model. Brazilian journal of medical and biological research $=$ Revista brasileira de pesquisas medicas e biologicas $/$ Sociedade Brasileira de Biofisica ... [et al.]. 33, 457-465

16 Rohr, S. (2004) Role of gap junctions in the propagation of the cardiac action potential. Cardiovasc Res. 62, 309-322

17 Figueroa, X. F. and Duling, B. R. (2009) Gap junctions in the control of vascular function. Antioxid Redox Signal. 11, 251-266

18 Kidder, G. M. and Mhawi, A. A. (2002) Gap junctions and ovarian folliculogenesis. Reproduction. 123, 613-620

19 Bruzzone R, D. R. (2006) Structure and functions of gap junctions in the developing brain. Cell Tissue Res. 326, 239-248

20 Campbell, F. R. (1980) Gap junctions between cells of bone marrow: an ultrastructural study using tannic acid. The Anatomical record. 196, 101-107

21 Dorshkind, K., Green, L., Godwin, A. and Fletcher, W. H. (1993) Connexin-43-type gap junctions mediate communication between bone marrow stromal cells. Blood. 82, 38-45

22 Oviedo-Orta, E., Hoy, T. and Evans, W. H. (2000) Intercellular communication in the immune system: differential expression of connexin 40 and 43 , and perturbation of gap junction channel functions in peripheral blood and tonsil human lymphocyte subpopulations. Immunology. 99, $578-590$

23 Oviedo-Orta, E. and Howard Evans, W. (2004) Gap junctions and connexin-mediated communication in the immune system. Biochimica et biophysica acta. 1662, 102-112

24 Oviedo-Orta, E., Errington, R. J. and Evans, W. H. (2002) Gap junction intercellular communication during lymphocyte transendothelial migration. Cell biology international. 26, 253-263

25 Wong, C. W., Christen, T., Roth, I., Chadjichristos, C. E., Derouette, J. P., Foglia, B. F., Chanson, M., Goodenough, D. A. and Kwak, B. R. (2006) Connexin37 protects against atherosclerosis by regulating monocyte adhesion. Nature medicine. 12, 950-954

26 Vaiyapuri, S., Jones, C. I., Sasikumar, P., Moraes, L. A., Munger, S. J., Wright, J. R., Ali, M. S., Sage, T., Kaiser, W. J., Tucker, K. L., Stain, C. J., Bye, A. P., Jones, S., Oviedo-Orta, E., Simon, A. M., Mahaut-Smith, M. P. and Gibbins, J. M. (2012) Gap junctions and connexin hemichannels underpin hemostasis and thrombosis. Circulation. 125, 2479-2491

27 Angelillo-Scherrer, A., Fontana, P., Burnier, L., Roth, I., Sugamele, R., Brisset, A., Morel, S., Nolli, S., Sutter, E., Chassot, A., Capron, C., Borgel, D., Saller, F., Chanson, M. and Kwak, B. R. (2011) Connexin 37 limits thrombus propensity by downregulating platelet reactivity. Circulation. 124, 930-939

28 Cancelas, J. A., Koevoet, W. L., de Koning, A. E., Mayen, A. E., Rombouts, E. J. and Ploemacher, R. E. (2000) Connexin-43 gap junctions are involved in multiconnexin-expressing stromal support of hemopoietic progenitors and stem cells. Blood. 96, 498-505

29 Krenacs, T. and Rosendaal, M. (1998) Connexin43 gap junctions in normal, regenerating, and cultured mouse bone marrow and in human leukemias: their possible involvement in blood formation. The American journal of pathology. 152, 993-1004

30 Ploemacher, R. E., Mayen, A. E., De Koning, A. E., Krenacs, T. and Rosendaal, M. (2000) Hematopoiesis: Gap Junction Intercellular Communication is Likely to be Involved in Regulation of Stroma-dependent Proliferation of Hemopoietic Stem Cells. Hematology. 5, 133-147

31 Vaiyapuri, S., Moraes, L. A., Sage, T., Ali, M. S., Lewis, K. R., Mahaut-Smith, M. P., Oviedo-Orta, E., Simon, A. M. and Gibbins, J. M. (2013) Connexin40 regulates platelet function. Nature communications. 4, 2564 
32 Harris, A. L. (2001) Emerging issues of connexin channels: biophysics fills the gap. Quarterly reviews of biophysics. 34, 325-472

33 Giepmans, B. N. (2004) Gap junctions and connexin-interacting proteins. Cardiovascular research. 62, 233-245

34 Burger, P. C. and Wagner, D. D. (2003) Platelet P-selectin facilitates atherosclerotic lesion development. Blood. 101, 2661-2666

35 Massberg, S., Schurzinger, K., Lorenz, M., Konrad, I., Schulz, C., Plesnila, N., Kennerknecht, E., Rudelius, M., Sauer, S., Braun, S., Kremmer, E., Emambokus, N. R., Frampton, J. and Gawaz, M. (2005) Platelet adhesion via glycoprotein IIb integrin is critical for atheroprogression and focal cerebral ischemia: an in vivo study in mice lacking glycoprotein IIb. Circulation. 112, 1180-1188

\section{Figure Legends}

Figure 1: Connexins, hemichannels and gap junctions. This image depicts the architecture of connexins with four transmembrane domains and extracellular and intracellular loop regions. Six connexin monomers oligomerise to form a hemichannel which facilitates the transport of signalling molecules between the cytoplasm and external environment. Upon docking, two hemichannels from adjacent cells form a gap junction to allow intercellular communication.

Figure 2: Gap junctions between platelets. Presence of gap junction-like structures between platelets was explored by analysing agonist-induced platelet aggregates using transmission electron microscopy. The arrows indicate the gap junction-like structures. The inner subsets show the enlarged regions of gap junction-like structures. This image is from reference [26].

Figure 3: Connexin hemichannels and gap junctions regulate thrombosis. The effect of connexin mimetic peptide, ${ }^{37,43} \mathrm{Gap} 27$ on thrombosis was analysed in mice using intravital microscopy. Platelets were labelled with an Alexa fluor 488 conjugated anti-GPIb antibody and thrombus formation was induced using an ablation laser in cremaster muscle arterioles. The images shown (A) are representative of thrombi obtained at different time intervals. The thrombus intensity was quantified to compare the effect of connexin inhibitor with the control (scrambled peptide) $(p=<0.05)$ (B). This image is from reference [26]. 
Figure 1

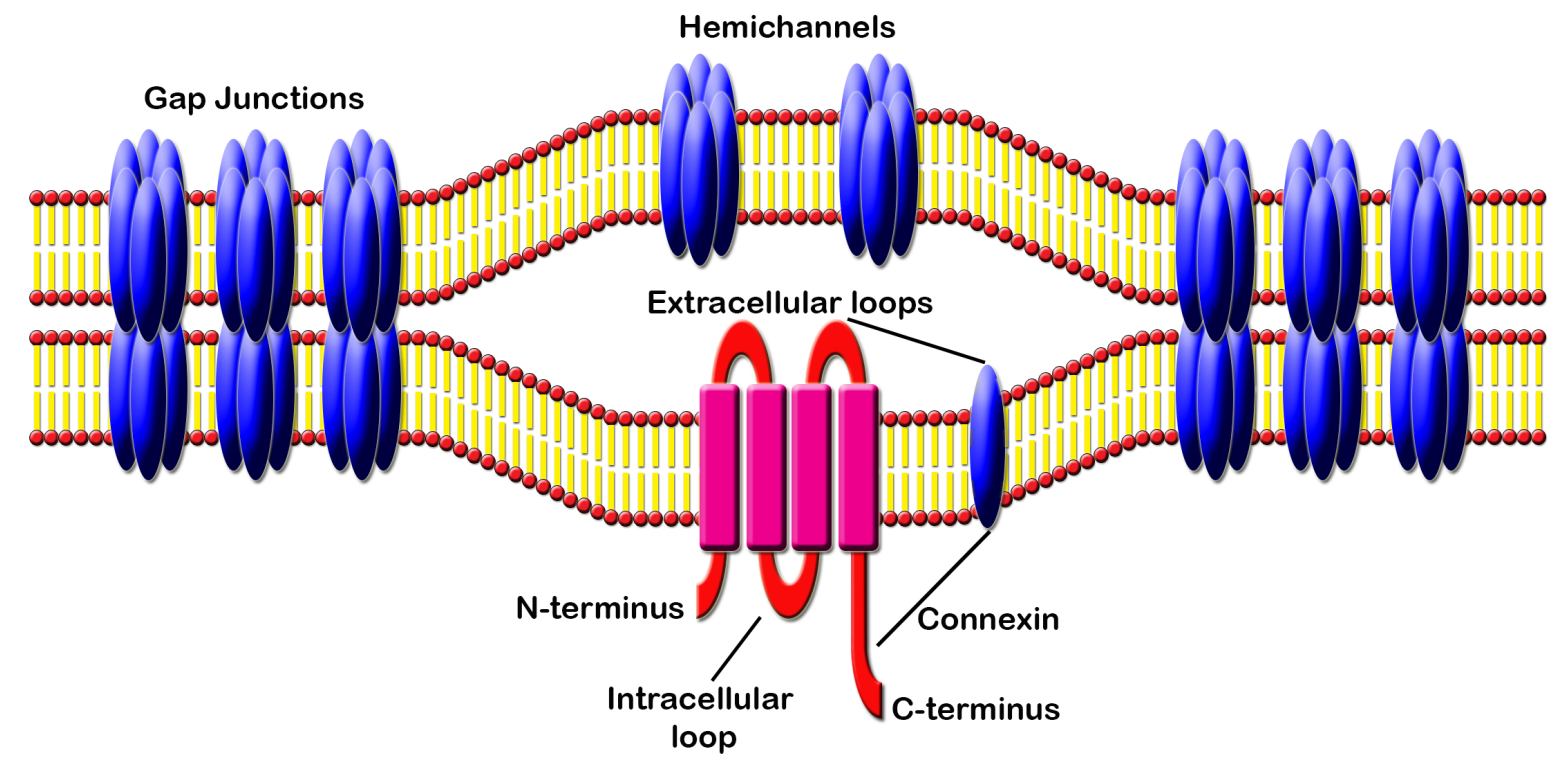


Figure 2
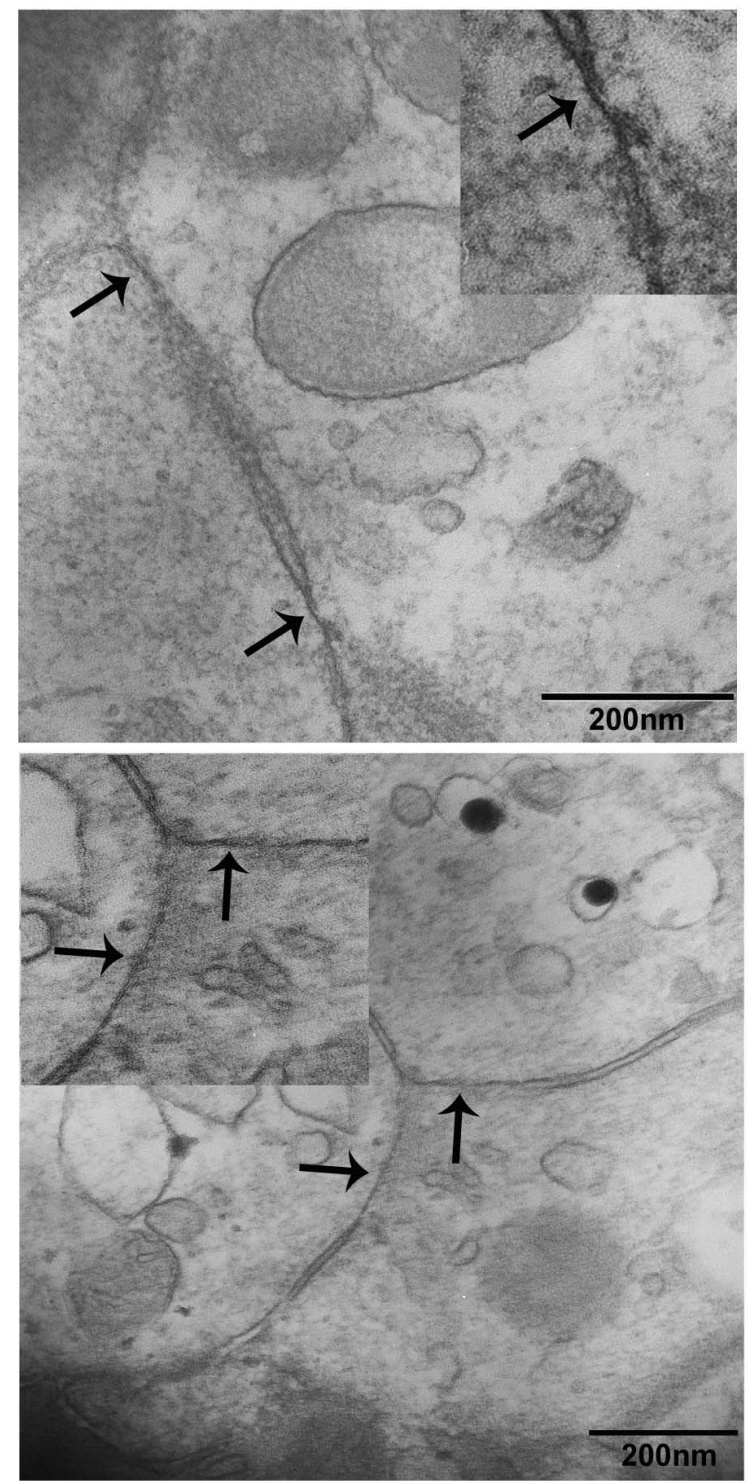
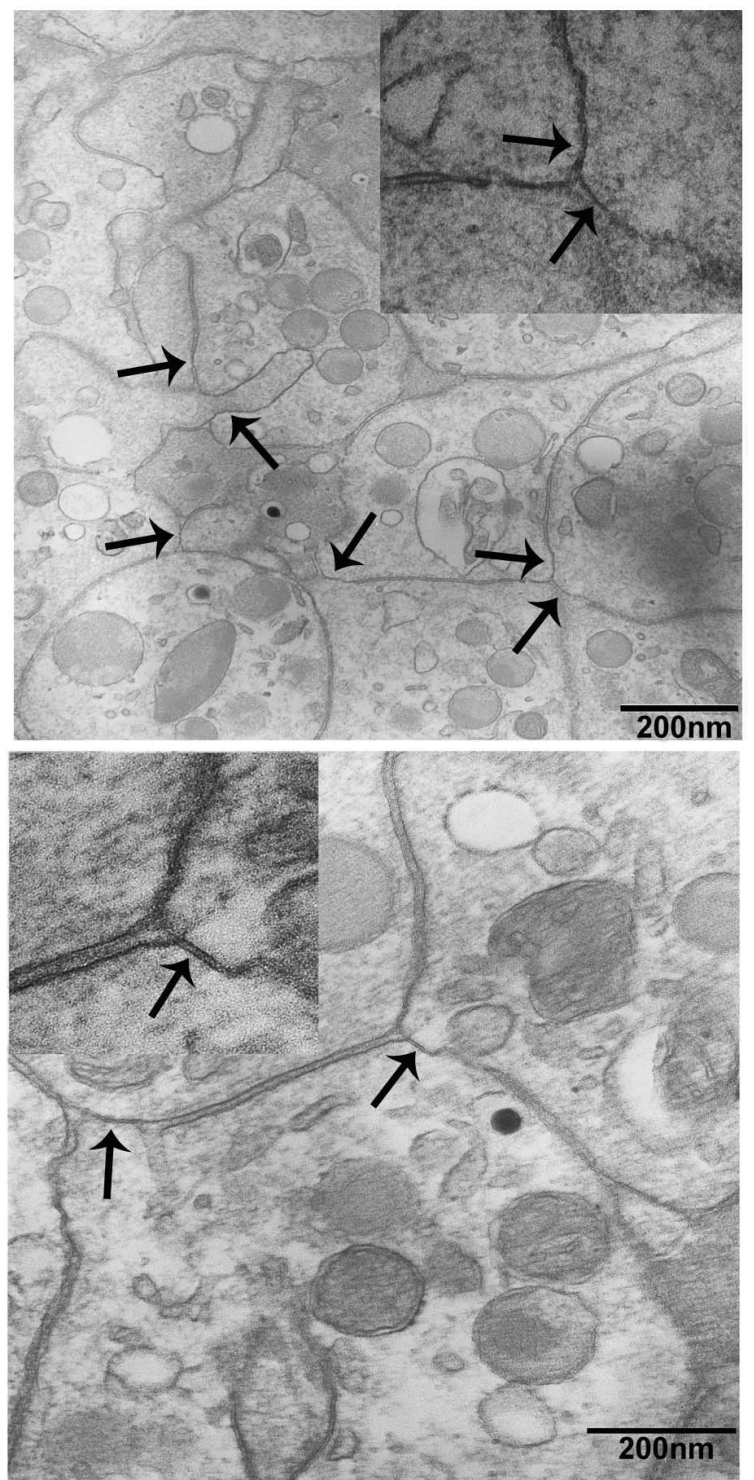
Figure 3

A

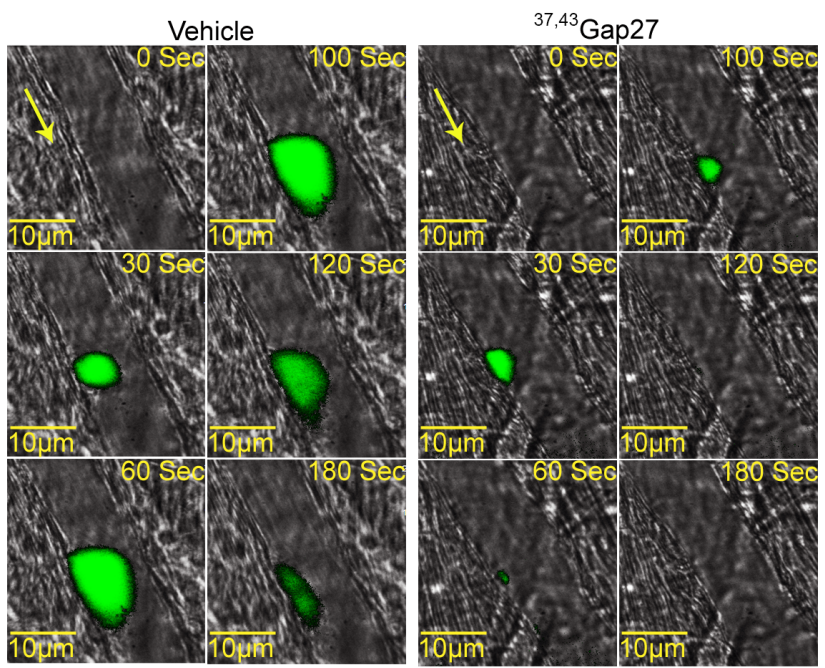

B

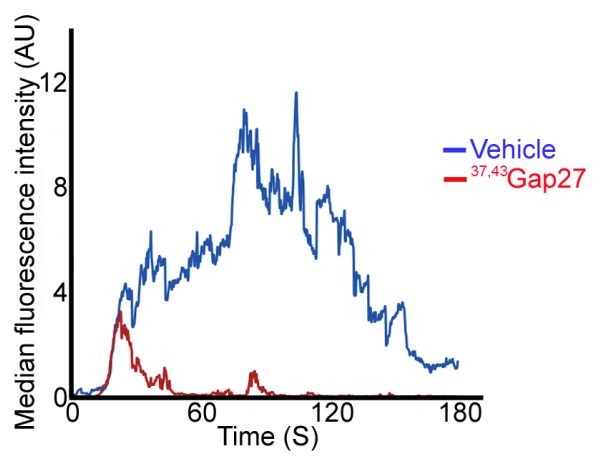

